\title{
Almost Nothing - Not Even Bariatric Surgery for Obesity - Changes Olfactory Sensitivity
}

\author{
Paul Enck, Nicole Rieber, Helene Sauer, Sibylle Klosterhalfen, Isabelle Mack, \\ Stephan Zipfel and Martin Teufel
}

University Medical Hospital, Dept. of Psychosomatic Medicine, Frondsbergstr. 23, 72076 Tübingen, Germany

Correspondence should be addressed to: Paul Enck; paul.enck@uni-tuebingen.de

Received date: 1 December 2013; Accepted date: 9 January 2014; Published date: 28 February 2014

Academic Editor: Virginia Quick

Copyright @ 2014. Paul Enck, Nicole Rieber, Helene Sauer, Sibylle Klosterhalfen, Isabelle Mack, Stephan Zipfel and Martin Teufel. Distributed under Creative Commons CC-BY 3.0

\begin{abstract}
Altered olfactory functions in patients with eating disorders have been reported with obesity, during hunger, following food ingestion and after bariatric surgery, but subsequent data have remained controversial. Methods: We investigated olfactory function (threshold, identification, discrimination) by Sniffin Sticks ${ }^{\circledR}$ in six independent studies: At baseline and $24 \mathrm{~h}$ and $48 \mathrm{~h}$ after a zero-calorie fasting (Study 1); fasted and after a $3810 \mathrm{~kJ}$ mixed meal (Study 2); twice after a $2510 \mathrm{~kJ}$ mixed meal supplemented with either $40 \mathrm{~g}$ saturated or unsaturated fatty acids (Study 3); prior to and after rotation-induced nausea (Study 4); at baseline and after a 5-day $6^{\circ}$ head-down tilt bedrest (HDT-BR) and a high $(7.7 \mathrm{mmol} / \mathrm{kg}$ $\mathrm{BW} / \mathrm{d})$ and low $(0.7 \mathrm{mmol} / \mathrm{kg} \mathrm{BW} / \mathrm{d})$ sodium diet (Study 5). We also compared odor sensitivity before and 12 months after bariatric (sleeve) surgery in patients with morbid obesity (Study 6). Results: Fasting (Study 1) did not alter olfactory functions, and a highcalorie diet (Study 2) moderately improved odor discrimination only; saturated fatty acids (Study 3) marginally improved odor identification, while the induction of nausea (Study 4) did not affect olfaction. HDT-BR (Study 5) significantly reduced the olfactory thresholds as well as the total score, mainly due to the bedrest component. Bariatric surgery (Study 6) did not change odor sensitivity, but some morbidly obese patients exhibited abnormally low olfactory functions prior to and after surgery. Conclusions: Manipulation of the hunger/satiety state has only marginal effects on olfactory functions in normal healthy volunteers and obese patients.
\end{abstract}

Keywords: obesity, bariatric surgery, chemosensitivity, food, diet.

\section{Introduction}

Both smell and taste are important predigestive cues relevant for finding and identifying food and its palatability and toxicity (Rolls 2005); taste preference and aversion have been shown to be effective learning indicators in animals and humans
(Stehberg et al. 2011), especially relevant in species not able to eliminate toxic food after ingestion (Parker \& Limebeer 2006). Such predigestive signals also serve the preparation of the gastrointestinal tract by inducing secretory and motility responses (Davis \& Smith 2009) (called anticipatory 
or sham feeding responses), and have been proposed to be of pathophysiological relevance in patients with gastrointestinal diseases (Lunding et al. 2008), but also with eating disorders (anorexia, bulimia) (Rapps et al. 2007).

Altered odor sensitivity has been reported in patients with anorexia nervosa and bulimia (Rapps et al. 2008, Schreder et al. 2008), obesity (Naka et al. 2010), diabetes (Richardson et al. 2004), depression (Pause et al. 2001), schizophrenia (Turetsky et al. 2009), migraine (Pentzek et al. 2007), and Parkinson's disease (Bohnen et al. 2010), but also in the irritable bowel syndrome (Steinbach et al. 2010) and following intestinal surgery (Harris \& Griffin 2003). Altered taste sensitivity has been found following bariatric surgery (Pepino et al. 2013), while olfaction has not been investigated so far. These chemosensitivity changes have been attributed to altered central processing of sensory signals, e.g. controlled by food-ingestion controlling hormones such as Ghrelin (Tong et al. 2011), but also due to genetic mutations (Hasin-Brumshtein et al. 2009).

Altered olfactory functions during hunger, fasting episodes, and following food ingestion in healthy subjects have also been reported as early as 1949 (Janowitz and Grossman 1949), but data have remained controversial, probably not only due to the different methodologies to assess olfactory functions, but also due to differences in experimental protocols, e.g. duration of fasting, types of food ingested, timing of assessment, etc.

We wondered whether experimentally induced modulations of the digestive state are able to change the ability to sense, identify and discriminate standardized odor cues in healthy male and female subjects. As our overall research focus is on the psychobiology of eating, eating disorders, and the regulation of intestinal functions, we added a test of olfactory sensitivity to a series of independent experiments we conducted in the past 5 years. We tested the effects of prolonged (48h) zero-calorie fasting, high caloric $(3810 \mathrm{~kJ})$ food intake, high (40 g) fatty acid ingestion, rotation-induced nausea, and prolonged (5-day) head-down tilt (HDT) bedrest with high/low salt diet on olfactory function using a standardized and validated odor test (Sniffin Sticksß) (Kobal et al. 2000, Hummel et al. 2007) in healthy volunteers. We also compared odor sensitivity before and after bariatric surgery in patients with morbid obesity.

Our basic hypotheses were as follow: Upregulating of hunger by fasting should increase odor sensitivity, while downregulating it (from regular to strong satiety after meals of different caloric load) should decrease odor sensitivity. Inducing nausea by a non-food mechanism (rotation) should also reduce odor sensitivity. We expected olfactory sensitivity to decrease also with HDT, but we did not have a directed hypothesis regarding the effects' high/low sodium intake on olfaction. We expected olfactory sensitivity to be increased in morbid obesity and to return to normal after bariatric surgery.

\section{Methods}

The experiments were carried out at different locations in Tübingen and Cologne, Germany, but all under supervision of the principle investigator between 2004 and 2010. All volunteers participated in one experiment only, and gave written informed consent prior to participation. Participants were collected via advertisement on blackboards and newsletters, and received financial compensation for participation: for studies 1 to 4 , they received on average $15 €$ per hour testing; in the HDT bedrest study (Study 5), they received a total compensation of approximately 100 Euros per day for the full 2 x 21 day study; and in study 6 patients received no honorarium but were reimbursed for travel expenses. All people had been screened to exclude any acute or chronic disease or medication intake except contraceptives in female subjects. We specifically screened and excluded those with increased scores on psychometric tests (e.g. the German version of the Patient Health Questionnaire, PHQ). All protocols had been approved by the Ethical Committee of the University of Tübingen Medical Faculty, except the bedrest study that was approved by the Ethics Board of the Physicians' Chamber (Ärztekammer) in North-Rhine-Westphalia, Cologne, Germany. 
In most cases, the data reported here represent a small subset of all data collected (the fasting study, the fatty acid study, the HDT study); in others (food study, nausea study) the experiments were performed to test olfactory sensitivity mainly. The clinical study is also part of a larger investigation on physiological and psychological consequences of bariatric surgery in morbid obese patients (Rieber et al. 2012, Teufel et al. 2012).

In all investigation, odor sensitivity was tested using the same standard test (Sniffin` Sticks) (Kobal et al. 2000, Hummel et al. 2007). All investigations were crossover studies investigating subjects twice or more under experiment, and under a control condition. The patient study was a repeated test of olfactory sensitivity prior to and 12 months after stomach surgery.

\section{Olfactory Sensitivity Testing}

The "Sniffin` Sticks $₫$ " test (Burghart Medizintechnik GmbH, Wedel, Germany) consists of the three subtests: phenyl ethyl alcohol odor threshold, discrimination, and identification (for validation of the test see (Kobal et al. 2000, Hummel et al. 2007)). This test has also been validated in a number of clinical investigations, also in patients with eating disorders (Rapps et al. 2010, Schreder et al. 2008, Simchen et al. 2006, Aschenbrenner et al. 2008, Roessner et al. 2005, Fedoroff et al. 1995).

For the odor threshold test, three pens were presented simultaneously with one only containing one odorant in a certain dilution, while the other two contained the solvent. The subjects had to identify the odor-containing pen. Triplets with increasing concentrations were presented, and the staircase was reversed with correct identification. Threshold was defined as the mean of the last four out of seven staircase reversal points. The scores ranged between 1 and 16, and a higher score indicates higher sensitivity.

The odor discrimination test was performed by means of triplets of odorants - two pens containing the same odorant and the third a different odorant. Odorants comprising a triplet were similar with regard to intensity and hedonic tone. The subject's task was to identify, which of the three odor-containing pens smelled different. With a total of 16 triplets tested, the scores ranged again from 0 to 16 , and a higher score indicates a better ability of odor discrimination.

For the odor identification, the test presented 16 common odorants like peppermint, vanilla, etc. The criteria for the selection of odorants were similar as described for the odor discrimination test, but subjects had to choose from 4 possible answers presented. Subjects were free to sample the odors as often as necessary to take a decision. The interval between presenting different odor identification tasks was at least $30 \mathrm{~s}$ to prevent olfactory desensitization. The scores ranged from 0 to 16 (1 point for each successful answer), higher scores indicating a better ability of odor identification.

The sum of results of the three Sniffin' Sticks subtests is presented as TDI score. Previous work has established the testretest reliability and its repetitive use in experimental setting (Kobal et al. 2000, Hummel et al. 2007).

\section{The Experimental Protocols}

Table 1 summarizes the major characteristics of the studies and the participants included. 
Table 1: Sample characteristics and study designs

\begin{tabular}{|c|c|c|c|c|c|c|}
\hline & \multicolumn{6}{|l|}{ Study } \\
\hline & $\begin{array}{l}1: \\
\text { Fasting }\end{array}$ & $\begin{array}{l}2: \\
\text { Food }\end{array}$ & \begin{tabular}{|l|} 
3: \\
Fatty acid \\
\end{tabular} & $\begin{array}{l}4: \\
\text { Nausea }\end{array}$ & $\begin{array}{l}\text { 5: } \\
\text { Bedrest }\end{array}$ & 6: Surgery \\
\hline $\mathbf{N}$ & 16 & 20 & 24 & 44 & 8 & 8 \\
\hline Age & $21.4 \pm 2.1$ & $24.6 \pm 2.0$ & $24.6 \pm 1.9$ & $24.7 \pm 1.9$ & $26.4 \pm 3.9$ & $47.6 \pm 11.4$ \\
\hline F:M & $16: 0$ & $0: 20$ & $12: 12$ & $22: 22$ & $0: 8$ & $4: 4$ \\
\hline Measures & 3 & 2 & 4 & 2 & 4 & 2 \\
\hline Design & Repeated & Cross-over & Cross-over & Cross-over & Cross-over & Repeated \\
\hline Control & baseline & baseline & placebo & baseline & baseline & baseline \\
\hline Intervention & $\begin{array}{l}24 / 48 \mathrm{~h} \\
\text { zero-calorie } \\
\text { fasting }\end{array}$ & $\begin{array}{l}\text { standard } \\
\text { meal, } \\
3800 \mathrm{~kJ}\end{array}$ & $\begin{array}{lr}500 \mathrm{mg} & 5- \\
\text { HTP/ } & \text { fatty } \\
\text { acids, } & 2500 \\
\text { kJ } & \\
\end{array}$ & $\begin{array}{l}\text { body rotation } \\
\text { for } 5 \times 2 \mathrm{~min}\end{array}$ & $\begin{array}{l}\text { bedrest }+ \\
\text { high or low } \\
\text { salt content }\end{array}$ & $\begin{array}{ll}\text { pre } & +12 \\
\text { months } & \text { post } \\
\text { surgery } & \end{array}$ \\
\hline
\end{tabular}

N: Number of participants included; F: Female, M: Male

Fasting Study: Sixteen healthy female volunteers $(21.4 \pm 2.1$ years $)$ with normal body-mass index (BMI) $\left(>19 \mathrm{~kg} / \mathrm{m}^{2}\right)$ were investigated at baseline (within 3 hours after a regular breakfast at home), and $24 \mathrm{~h}$ and $48 \mathrm{~h}$ later after staying fasted on a zero-calorie diet with water ad lib, while on a metabolic ward under strict compliance control (Enck et al. 2009). The volunteers were involved in a series of experiments and tests with respect to autonomic functions (heart-rate variability), energy expenditure at rest and during cycling, and neurocognitive tests following food signals (eye-tracking). All olfactory tests were done at the same time of the days $(10.00 \mathrm{~h})$ and prior to the other tests.

Food Study: Twenty healthy male subjects (24.6 \pm 2.0 years, normal BMI) were investigated twice, after an overnight fast at $8.00 \mathrm{~h}$ in the morning and 15 minutes after a standardized meal (pizza baguette, ice cream, apple juice) (3810 kJ), $100 \mathrm{~g}$ carbohydrates, $38 \mathrm{~g}$ fat, $21 \mathrm{~g}$ protein) at $12.00 \mathrm{~h}$. The sequences of test days were randomized but balanced, and the tests were at least 3 days apart.

Fatty Acid Study: Twenty-four subjects (12 females) (24.6 \pm 1.9 years) were investigated four times, with at least 3 days in-between two tests, and on each day 3 hours after a standard mixed meal containing $40 \mathrm{~g}$ of either predominantly saturated or unsaturated fatty acids (olive oil or butter, resp.) (2546 kJ and $2303 \mathrm{~kJ}$ resp) (32 g carbohydrates, 12 g protein), plus 5-hydroxytryptophan (500 $\mathrm{mg})$ or placebo (Sauer et al. 200). We here report only the data for the unsaturated fatty acid meal (baseline), and the saturated fatty acid meal under placebo condition. The sequences of tests were randomized but balanced.

Nausea Study: Forty-four healthy subjects (22 females) (24.7 \pm 1.9 years) were investigated twice, under baseline conditions after a $3 \mathrm{~h}$ fast and within 30 minutes, after they had been rotated along the body axis in a conventional rotation chair for 5 x 90 sec (with 1 min interruptions in-between runs), with nausea-enforcing head movements along the yaw-axis every $6 \mathrm{sec}$, as previously described (Rieber et al. 2010).

Bedrest Study: Eight healthy male subjects (26.4 \pm 3.9 years) were investigated at two occasions months apart. At both occasion, they were tested immediately prior to HDT bedrest (baseline) on a balanced control $\operatorname{diet}(2.8 \mathrm{mmol}$ sodium $/ \mathrm{kg} \mathrm{BW} / \mathrm{d}$ ), and after 5 days (of a total of 21 days) of 6 degree HDT bedrest, on either a low sodium $(0.7$ $\mathrm{mmol} / \mathrm{kg} \mathrm{BW} / \mathrm{d}$ ) or a high sodium diet (7.7 $\mathrm{mmol} / \mathrm{kg} \mathrm{BW} / \mathrm{d}$ ) in randomized sequence, with diet adjusted to the individual body weight. The study was conducted at the German Aerospace Research Center (DLR) Cologne, Germany (Frings-Meuthen et al. 2011).

Bariatric Study: Eight morbidly obese patients ( $47.6 \pm 11.4$ years, 4 females, BMI: $47.9 \pm 6.5$ prior to surgery) were tested prior to sleeve gastrectomy (Nadler et al. 2012), and 12 months post surgery for a 
number of psychological and physiological functions (Rieber et al. 2012, Teufel et al. 2012).

\section{Statistics}

Sensory thresholds for odor perception, odor discrimination and identification, and total scores of olfactory sensitivity (called TDI score) were compared between conditions by repeated measure ANOVA with the level of significance set at 5\%. Correlations between the two measures were calculated by Pearson's correlation coefficient. Data were also compared to the age-adjusted standard norms of the test. All analyses were performed with the SPSS Ver 13 statistical program.

\section{Results}

Fasting Study: None of the measures of odor sensitivity were altered after $48 \mathrm{~h}$ of fasting compared to baseline (Table 2). All values were well within the normal range before and after fasting (Figure 1), but tended $(p<.10)$ to increase following fasting. Data after 24 hours of fasting were not different (data not shown).

Table 2: Olfactory characteristics (threshold, identification, discrimination, compound measure of all three=TDI) at baseline and post-intervention. Significant intervention effects $(p<.05)$ are printed in bold. All data are within the normal values reported for the test (21).

\begin{tabular}{|l|l|l|l|l|l|l|}
\hline & \multicolumn{6}{|l|}{ Study } \\
& $\begin{array}{l}1: \\
\text { Fasting* }\end{array}$ & $\begin{array}{l}2: \\
\text { Food }\end{array}$ & $\begin{array}{l}\text { 3: } \\
\text { Fatty acid }\end{array}$ & $\begin{array}{l}4: \\
\text { Nausea }\end{array}$ & $\begin{array}{l}5: \\
\text { Bedrest }^{*}\end{array}$ & 6: Surgery $^{\circ}$ \\
\hline Baseline & & & & & & \\
\hline Threshold & $9.2 \pm 1.5$ & $8.0 \pm 1.9$ & $8.9 \pm 2.9$ & $8.7 \pm 2.2$ & $10.1 \pm 1.1$ & $5.9 \pm 1.8$ \\
\hline Identification & $13.8 \pm 1.2$ & $12.9 \pm 1.6$ & $13.3 \pm 1.9$ & $13.2 \pm 1.3$ & $14.1 \pm 1.4$ & $9.8 \pm 1.4$ \\
\hline Discrimination & $12.9 \pm 2.0$ & $12.5 \pm 1.8$ & $12.8 \pm 1.9$ & $13.7 \pm 1.5$ & $13.0 \pm 1.6$ & $11.3 \pm 3.2$ \\
\hline TDI & $35.8 \pm 3.4$ & $33.4 \pm 3.4$ & $35.0 \pm 5.5$ & $35.2 \pm 3.7$ & $37.3 \pm 3.5$ & $26.9 \pm 6.0$ \\
\hline Intervention & & & & & & \\
\hline Threshold & $8.9 \pm 1.3$ & $8.0 \pm 1.5$ & $8.2 \pm 2.7$ & $9.0 \pm 2.1$ & $\mathbf{8 . 7} \pm \mathbf{1 . 8}$ & $5.8 \pm 2.0$ \\
\hline Identification & $14.2 \pm 1.6$ & $13.0 \pm 1.9$ & $\mathbf{1 3 . 8} \pm \mathbf{1 . 7}$ & $13.3 \pm 1.2$ & $13.8 \pm 1.2$ & $9.6 \pm 2.6$ \\
\hline Discrimination & $13.8 \pm 1.3$ & $\mathbf{1 3 . 2} \pm \mathbf{1 . 9}$ & $12.8 \pm 2.7$ & $13.3 \pm 2.6$ & $11.9 \pm 1.6$ & $12.1 \pm 2.2$ \\
\hline TDI & $37.1 \pm 2.7$ & $34.1 \pm 3.5$ & $34.7 \pm 6.2$ & $35.6 \pm 3.7$ & $\mathbf{3 4 . 3} \pm \mathbf{2 . 3}$ & $27.6 \pm 5.3$ \\
\hline
\end{tabular}

*: baseline and post $48 \mathrm{~h}$ only

+: placebo/unsaturated vs placebo/saturated fatty acid only

\#: baseline and bedrest/low salt only

${ }^{\circ}$ : pre and 12 months post surgery

Food study: Ingestion of a standard 3810 $\mathrm{kJ}$ meal did not change the perception of odor stimuli (Table 2), except that odor discrimination moderately improved $(F=4.65, p=.044)$, but remained within normal ranges pre and post food challenge. All values were well within the normal range at baseline and after eating.

Fatty Acid Study: Ingestion of high loads of saturated or unsaturated fatty acids did induce high levels of satiety, and even nausea in some cases, but did not change chemosensation (Table 2), except that odor identification moderately increased ( $F=4.47, p=.045)$ with saturated fatty acids, as compared to the meal with predominantly unsaturated fatty acids. However, data under both conditions remained within the normal range. One male subject clearly exhibited signs of anosmia (TDI values < 15) under both conditions, while another subject was very sensitive (TDI > 40) (see Figure 1). 


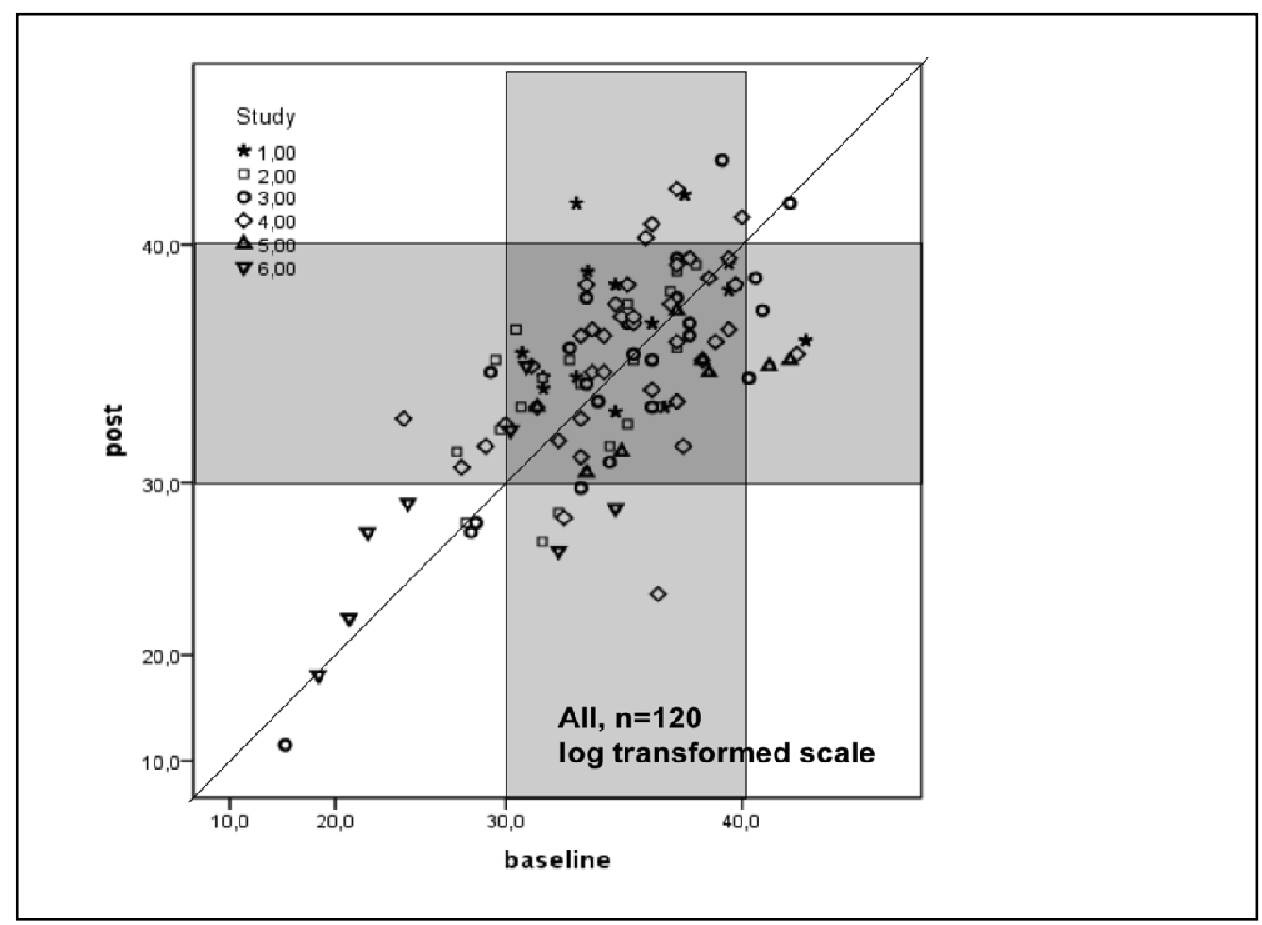

Figure 1: Scatter plot of baseline and post-intervention measures of olfactory sensitivity (TDI score) in 120 volunteers and patients in 6 different studies (see text for details). The shaded area indicates the range of normal values for the text. Except for study 6 (bariatric surgery), most values were within the normal range at both occasions, and their assembly around the $45^{\circ}$-regression line indicates minor changes between baseline and post-intervention values.

Nausea Study: While inducing nausea via body rotation significantly reduces hunger (data not shown), it did not alter the ability to perceive odor signals that remained within normal ranges (Table 2).

Bedrest Study: HDT bedrest significantly reduced the ability to perceive odors (TDI score: $F=9.36, p=.018$ ), mainly due to the decrease in discrimination $(F=10.02$, $\mathrm{p}=.016$ ) (Figure 2), while the discrimination capacity was reduced by the salt content of the diet (interaction Diet $\mathrm{x}$ Bedrest, $F=5.73, p=.048$ ). 


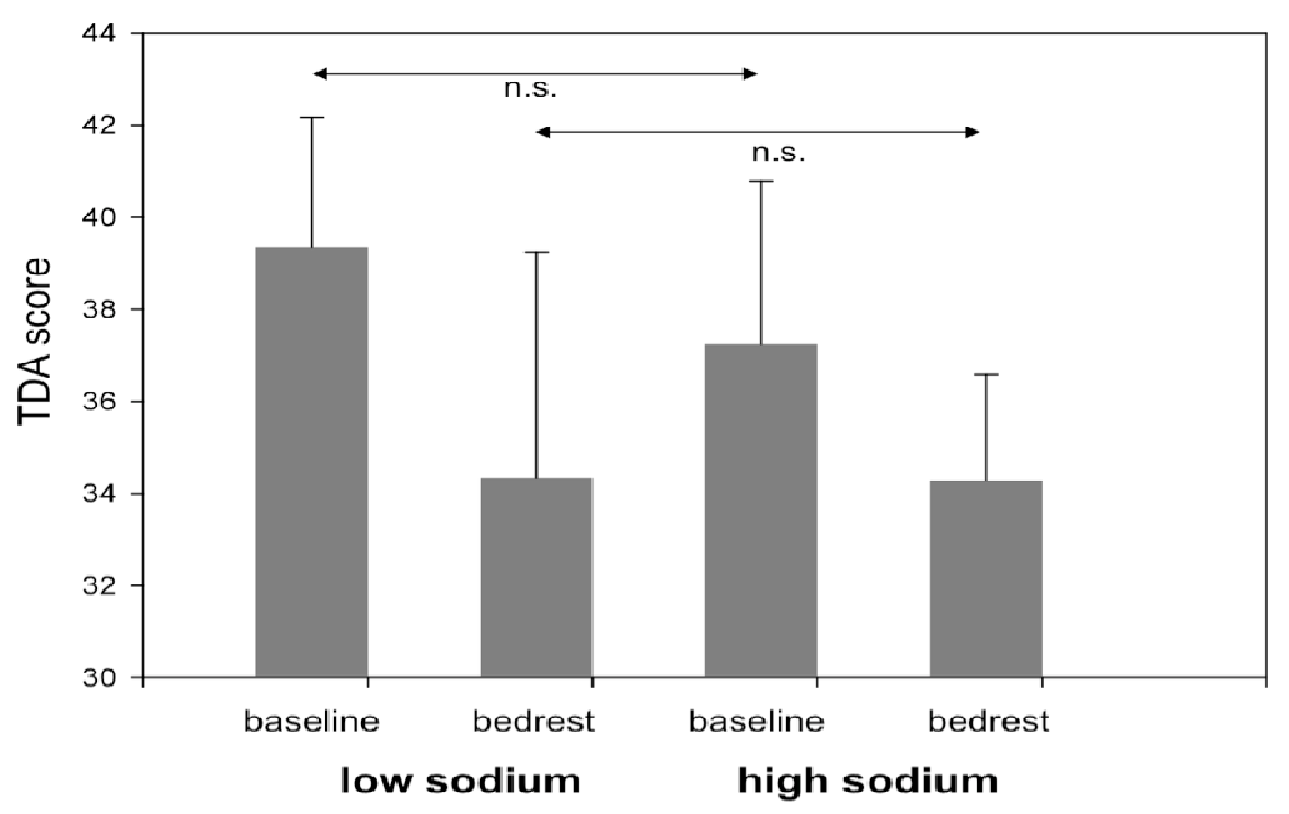

Figure 2: Olfactory sensitivity (TDI score) in Study 5 (head-down tilt bedrest) during two baseline measures and after 5 days on a low and a high-sodium diet (see text for details). A 2 × 2 repeated measures ANOVA revealed a significant effect of bedrest only $(F=9.36$, $p=.018)$. The figure reports post-hoc t-test values.

Bariatric Study: Odor perception was not changed in patients that underwent sleeve gastrectomy despite substantial alterations in eating behaviors that resulted in weight loss of $46.1 \pm 12.0 \mathrm{~kg}$. As is shown in Figure 1 , three of the male patients and one female patient showed abnormally reduced olfactory sensitivity prior to and after surgery. In two more females, the normal perception prior to surgery was lost post surgery. These changes were not explained by the higher age of the patients in comparison to the volunteer studies (see Table 2).

Both body weight prior to surgery and body weight loss were not associated with altered odor perception thresholds when adjusted for age and gender (data not shown).

\section{Discussion}

Altered odor sensitivity has been reported in patients with eating (Rapps et al. 2010, Schredert et al. 2008) and metabolic disorders (Naka et al. 2010, Richardson et al. 2004), in psychiatric patients (Pause et al. 2001, Turetsky et al. 2009), patients with neurological diseases (Pentzek et al. 2007, Bohnen et al. 2010) and intestinal disorders and conditions (Steinbach et al. 2010, Harris and Griffin 2003). It has been attributed to hormonal (Tong et al. 2011) or genetic (Hasin-Brumshtein et al. 2009) control, but it was noted that altered sensory perception in anorexia nervosa for instance improved with improvement of body weight (Aschenbrenner et al. 2008) and thus is susceptible to short-term changes.

Occasional reports of altered olfactory functions during hunger, fasting episodes, and following food ingestion in healthy subjects (Janowitz and Grossman 1949, Stafford and Welbeck 2011, Albrecht et al. 2009, Koelega 1994, Yeomans and Mobini 2006, Pollatos et al. 2007) have remained controversial, most likely due to methodologies' difficulties, but also due to differences in experimental protocols. It has also been noted that cognitions and emotions may alter odor perception (Janowitz and Grossman 1949, Chen and 
Dalton 2005, Hedner et al. 2010), making the comparison of studies even more difficult.

In the series of studies reported here, we tested (among others) whether experimental modulation of the fasting/fed state in healthy volunteers in five experiments and following surgical removal of part of the stomach in obese patients would affect the sensitivity of the olfactory system in a predictable way: we would have expected to see decreased thresholds and increased identification and discrimination ability during hunger, and the reverse effects following food ingestion, especially with a high fatty acid load. We also expected olfactory sensitivity to decrease with a non-food induction of nausea since nausea reduces the desire to eat, and during simulation of zero-gravity via HDT bedrest, while we did not have a directed hypothesis with respect to the salt load of food during HDT bedrest. Finally, we expected that bariatric surgery would reduce olfactory sensitivity, as has been shown for taste sensitivity in the literature (Miras and le Roux 2010).

We were surprised to find that most of our hypothesis failed: Fasting does not affect the sensory perception of olfactory cues, neither does satiety nor nausea, and bariatric surgery had no effects even in those subjects that had abnormal thresholds for olfaction prior to surgery. Only with 5 days HDT bedrest, we found a significant increase in odor threshold due to the HDT component, and reduced odor identification abilities due to the high salt content of the diet.

The obvious discrepancy between the clinical finding of altered sensory perception in a number of eating and digestion-associated diseases as well as in centrally-mediated medical conditions on the one hand, and the missing action of respective experimental/clinical manipulations of the digestive state and physiology on olfaction calls for two possible explanations: Either the experimental manipulations are too moderate to mimic any of the central changes responsible for altered olfactory processing in those clinical conditions, or the tools for testing olfactory sensitivity are insensitive to catch any of the experimentally-induced changes in olfaction, but are merely able to identify alterations of smell sensitivity with severely altered clinical conditions.

This dilemma is not unknown: Differences in taste and smell perception have been reported between men and women (Doy and Cameron 2009), during the menstrual cycle (Navarrete-Palacios et al. 2003), and during pregnancy and (Ochsenbein-Kölble et al. 2007) and have been linked to specific taste aversions and taste preferences in pregnant women (Nordin et al. 2004), but have not been found to be associated with nausea during early pregnancy (Hummel et al. 2002). The mechanisms behind these phenomena, thus, are incompletely understood. Similarly, claimed higher odor sensitivity in "multiple chemical hypersensitivity" (Nordin et al. 2005) and in the "sickbuilding syndrome" (Wang et al. 2008) has been difficult to prove with currently available test tools. Whether methodological limitations of current test tools may be responsible for the missing efficacy of our experimental interventions on olfaction in our test series in comparison the clinical finding will only be answered when other technologies to test chemosensory functions (e.g. brain imaging (Wiesman et al. 2008)) can be used in larger series of patient and subjects.

Two of our findings are worth further consideration: one is the decreased olfactory sensitivity in subjects after 5-day HDT bedrest, and on a low/high sodium diet. Prolonged 6-degree HDT is an approved earth-bound experimental technique to simulate zero-gravity conditions such as during space flights (Trappe et al. 2001). It has been shown to alter bone metabolism (Trappe et al. 2001), cardiac functions (Trappe et al. 2006), and other physiological changes (Kanikowska et al. 2008) that have been observed in astronauts after long-term space flights, and thus can be studied in more detail under conventional laboratory conditions, e.g. during the 60-day HDT WISE study in women (Beller et al. 2011). Changes in olfactory capacity, especially higher sensory thresholds have occasionally been reported not only with commercial airflights (Burdack-Freitag et al. 2011) 
attributable to lowered cabin pressure, but also with space flights (Olabi et al. 2002). However, experimental data from simulation studies have remained controversial (Vickers et al. 2001, Mester et al. 1988). The mechanism behind this effect remains unclear but may be a consequence of an upward shift of body fluids, resulting, for example, in higher intracranial pressure or swelling of the mucosa tissue, although nasal airway resistance has been reported not to be changed with HDT bedrest (Steinbach et al. 2005). The effect appears to be stronger under the low salt diet for unknown reasons.

Contrary to previous but mostly anecdotal reports (Harris \& Griffin 2003), we could not substantiate findings that bariatric surgery - removal or reduction of a large proportion of the stomach - in morbidly obese subjects would affect chemosensitivity, at least for some taste components, e.g. sweets. In fact, half of our subjects had reduced smell sensitivity prior to surgery - which is compatible with the reported data from patients with metabolic disorders (Naka et al. 2010) - that did not improve; and in two cases chemosensitivity was worse 12 months after surgery but was normal before. Whether this represents the normal situation or an exception from the rule has to remain an open question until larger series of patients have been studied. As most reports are limited to sweet taste, this probably points towards a more cognitively controlled food (taste, smell) preferences and avoidances (Rieber et al. 2012, Teufel et al. 2012) than to physiological changes in chemosensitivity.

We need to acknowledge a number of limitations of our studies: Studies 1 to 4 are powered as pilot studies because the available data did not allow for proper power calculation; in Study 5, the case number was assessed based on metabolic data and logistics, possibilities at the research facility at DLR Cologne. We had to accept the time slots that were offered to us due to priorities beyond our control. However, access to such studies is a privilege as they are rarely done. Study 6 was part of an ongoing clinical trial and suffered from poor adherence of patients for the physiological studies. While we used the same olfactory test in all studies, the test stimuli decay over time (they can be used for a maximum of 3 months), and may therefore have affected the outcome in some studies; we used however, new test sets for each of the studies. We also included only males in some studies (Studies 2 and 5) and females in others (Study 1), despite known differences (Doy and Cameron 2009) for practical reasons, and any generalization of the data may be limited by this fact. When females were included, they were not recruited according to their menstrual cycle status known to have (limited) effects on chemosensitivity (Navarrete-Palacios et al. 2003), which may have obscured the results. Finally, we should have collected taste data in all studies but did only in a few, and therefore cannot conclude from olfactory sensitivity to chemosensitivity in general.

In summary however, olfactory sensitivity (threshold and identification and discrimination capacity) appear to be rather stable (hard-wired) chemosensory functions that respond poorly, if at all, to short and acute changes in food-related nutrient cues (both fasting and loading) and their processing, and even to chronic and persistent reduction of the stomachs' storage capacity. This is at odds with reported taste alterations following bariatric surgery, and with the role of taste and smell in a variety of eating disorders. It, also, is at odds with the reported recovery of pathologic odor perception in patients with anorexia nervosa after weight gain and warrants further investigations.

\section{Acknowledgement}

Supported by grants No. 1A-I, 7A-I, and 11A-II from the inter-university Center of Nutritional Medicine of the Universities of Tübingen and Hohenheim-Stuttgart, Germany. The funders had no role in study design, data collection and analysis, decision to publish, or preparation of the manuscript.

We would like to thank U.Kindler, U.Maier, L.Grossmann, A.Seifert, S.Kohler, and S.Siegle for whom the odor tests were part of their respective dissertational work. 
We also like to express our gratitude to Thomas Hummel, Dresden for logistic support and help with interpreting the data.

\section{References}

1. Albrecht, J., Schreder, T., Kleemann, A.M., Schöpf, V., Kopietz, R., Anzinger, A., Demmel, M., Linn, J., Kettenmann, B. and Wiesmann, M. (2009) "Olfactory detection thresholds and pleasantness of a foodrelated and a non-food odour in hunger and satiety." Rhinology, 47,160-165

2. Aschenbrenner, K., Scholze, N., Joraschky, P. and Hummel, T. (2008) "Gustatory and olfactory sensitivity in patients with anorexia and bulimia in the course of treatment." Journal of Psychiatric Research, 43,129-137.

3. Beller, G., Belavý, D.L., Sun, L., Armbrecht, G., Alexandre, C. and Felsenberg, D. (2011) "WISE-2005: bedrest induced changes in bone mineral density in women during 60 days simulated microgravity." Bone, 49, 858866.

4. Bohnen, N.I., Müller, M.L., Kotagal, V., Koeppe, R.A., Kilbourn, M.A., Albin, R.L. and Frey, K.A. (2010) "Olfactory dysfunction, central cholinergic integrity and cognitive impairment in Parkinson's disease." Brain, 133, 1747-1754.

5. Burdack-Freitag, A., Bullinger, D., Meyer, F. and Breuer, K. (2011) "Odor and taste perception at normal and low atmospheric pressure in a simulated aircraft." Journal of Consumer Protection and Food Safety, 6, 95109

6. Chen, D. and Dalton, P. (2005) "The effect of emotion and personality on olfactoryperception." Chemical Senses, 30, 345-351.

7. Davis, J.D. and Smith, G.P. (2009) "The conditioned satiating effect of orosensory stimuli." Physioogy \& Behavior, 97, 293303.

8. Doty, R.L. and Cameron, E.L. (2009) "Sex differences and reproductive hormone influences on human odor perception." Physiology \& Behavior, 97, 213-228.
9. Enck. P., Grau, F., Salini, A., Remmlinger, S., Günter, A., Haas, T., Giel, K.E., Rieber, N., Herbert, B.M., Zipfel, S., Otto, B. and Bischoff, S.C. (2009) "A 48-hour zero-calory diet significantly alters physiological and psychological functions." Zeitschrift für Gastroenterologie, 47, 914.

10.Fedoroff, I.C., Stoner, S.A., Andersen, A.E., Doty, R.L. and Rolls, B.J. (1995) "Olfactory dysfunction in anorexia and bulimia nervosa." International Journal of Eating Disorders, 18, 71-77.

11.Frings-Meuthen, P., Buehlmeier, J., Baecker, N., Stehle, P., Fimmers, R., May, F., Kluge, G., \& Heer, M. (2011). "High sodium chloride intake exacerbates immobilization-induced bone resorption and protein losses." Journal of Applied Physiology, 111, 537-542.

12.Harris, A.M. and Griffin, S.M. (2003) "Postoperative taste and smell deficit after upper gastrointestinal cancer surgery - an unreported complication." Journal of Surgical Oncology, 82, 147-150.

13.Hasin-Brumshtein, Y., Lancet, D. and Olender, T. (2009) "Human olfaction: from genomic variation to phenotypic diversity." Trends in Genetics, 25, 178-184

14.Hedner, M., Larsson, M., Arnold, N., Zucco, G.M. and Hummel, T. (2010) "Cognitive factors in odor detection, odor discrimination, and odor identification tasks." Journal of Clinical and Experimental Neuropsychology, 32, 1062-1067.

15.Hummel, T., von Mering, R., Huch, R. and Kölble, N. (2002) "Olfactory modulation of nausea during early pregnancy?" British Journal of Obstetrics and Gynaecology, 109, 1394-1397.

16.Hummel, T., Kobal, G., Gudziol, H. and Mackay-Sim, A. (2007) "Normative data for the "Sniffin' Sticks" including tests of odor identification, odor discrimination, and olfactory thresholds: an upgrade based on a group of more than 3,000 subjects." European Archives of Otorhinolaryngology, 264, 237-243.

17.Janowitz, H.D. and Grossman, M.I. (1949) "Gustoolfactory thresholds in relation to appetite and hunger sensations." Journal of Applied Physiology, 2, 217-222 
18.Kanikowska, D., Sato, M., Iwase, S., Shimizu, Y., Inukai, Y., Nishimura, N. and Sugenoya, J. (2008) "Immune and neuroendocrine responses to head-down rest and countermeasures." Aviation, Space, Environmental Medicine, 79, 1091-1095.

19.Kobal, G., Klimek, L., Wolfensberger, M., Gudziol, H., Temmel, A., Owen, C.M., Seeber, H., Pauli, E. and Hummel, T. (2000) "Multicenter investigation of 1,036 subjects using a standardized method for the assessment of olfactory function combining tests of odor identification, odor discrimination, and olfactory thresholds." European Archives of Otorhinolaryngology, 257, 205-211.

20.Koelega, H.S. (1994). "Diurnal variations in olfactory sensitivity and the relationship to food intake." Perception and Motor Skills, $78,215-226$.

21.Lunding, J.A., Nordström, L.M., Haukelid, A.O., Gilja, O.H., Berstad, A. and Hausken, T. (2008) "Vagal activation by sham feeding improves gastric motility in functional dyspepsia." Neurogastroenterology and Motility, 20, 618-624.

22.Mester, A.F., Doty, R.L., Shapiro, A. and Frye, R.E. (1988) "Influence of body tilt within the sagittal plane on odor identification performance." Aviation, Space, Environmental Medicine, 59, 734737.

23.Miras, A.D. and le Roux, C.W. (2010) "Bariatric surgery and taste: novel mechanisms of weight loss." Currunt Opinion in Gastroenterology, 26, 140-145.

24.Nadler, E.P., Barefoot, L.C. and Qureshi, F.G. (2012) "Early results after laparoscopic sleeve gastrectomy in adolescents with morbid obesity." Surgery, $152,212-217$.

25. Naka, A., Riedl, M., Luger, A., Hummel, T. and Mueller, C.A. (2010) "Clinical significance of smell and taste disorders in patients with diabetes mellitus." European Archives in Otorhinolaryngology, 267, 547550.

26.Navarrete-Palacios, E., Hudson, R., Reyes-Guerrero, G. and Guevara-Guzmán, R. (2003) "Lower olfactory threshold during the ovulatory phase of the menstrual cycle." Biological Psychology, 63,
269-279.

27.Nordin, S., Broman, D.A., Olofsson, J.K. and Wulff, M. (2004) "A longitudinal descriptive study of self-reported abnormal smell and taste perception in pregnant women." Chemical Senses, 29, 391-402.

28.Nordin, S., Martinkauppi, M., Olofsson, J., Hummel, T., Millqvist, E. and Bende, M. (2005) "Chemosensory perception and event-related potentials in self-reported chemical hypersensitivity." International Journal of Psychophysiology, 55, 243-255.

29.Ochsenbein-Kölble, N., von Mering, R., Zimmermann, R. and Hummel, T. (2007) "Changes in olfactory function in pregnancy and postpartum." International Journal of Gynaecology and Obstetrics, 97, 10-14.

30.Olabi, A.A., Lawless, H.T., Hunter, J.B., Levitsky, D.A. and Halpern, B.P. (2002) "The effect of microgravity and space flight on the chemical senses." Journal of Food Science, 67, 468-478.

31.Parker, L.A. and Limebeer, C.L. (2006) "Conditioned gaping in rats: a selective measure of nausea." Autonomic Neuroscience, 129, 36-41.

32.Pause, B.M., Miranda, A., Göder, R., Aldenhoff, J.B. and Ferstl, R. (2001) "Reduced olfactory performance in patients with major depression." Journal of Psychiatric Research, 35, 271-277.

33.Pepino, M.Y., Bradley, D., Eagon, J.C., Sullivan, S., Abumrad, N.A. and Klein, S. (2013) "Changes in taste perception and eating behavior after bariatric surgeryinduced weight loss in women." Obesity (Silver Spring) [in press]

34.Pentzek, M., Grass-Kapanke, B. and Ihl, R. (2007) "Odor identification in Alzheimer's disease and depression." Aging Clinical and Experimental Research, 19, 255-258.

35.Pollatos, O., Kopietz, R., Linn, J., Albrecht, J., Sakar, V., Anzinger, A., Schandry, R. and Wiesmann, M. (2007) "Emotional stimulation alters olfactory sensitivity and odor judgment." Chemical Senses, 32, 583589.

36.Rapps, N., Enck, P., Martens, U., Sammet, I., Teufel, M., Otto, B. and Zipfel, S. (2007) "[Digestive and predigestive functions in 
patients with eating disorders]." Zeitschrift für Gastroenterologie, 45:273-280.

37.Rapps, N., Giel, K.E., Söhngen, E., Salini, A., Enck, P., Bischoff, S.C. and Zipfel, S. (2010) "Olfactory deficits in patients with anorexia nervosa." European Eating Disorder Reviews, 18, 385-389

38.Rieber, N., Mischler, D., Schumacher, V., Muth, E.R., Bischoff, S.C., Klosterhalfen, S., Zipfel, S. and Enck, P. (2010) "Acute tryptophan depletion increases experimental nausea but also induces hunger in healthy female subjects." Neurogastroenterology and Motility, 22, 752-757.

39.Rieber, N., Giel, K.E., Meile, T., Enck, P., Zipfel, S. and Teufel, M. (2013) "Psychological dimensions after laparoscopic sleeve gastrectomy: reduced mental burden, improved eating behavior, and ongoing need for cognitive eating control." Surgery of Obesity and Related Disorders, 6, 569-573

40.Richardson, B.E., Vander Woude, E.A., Sudan, R., Thompson, J.S. and Leopold, D.A. (2004) "Altered olfactory acuity in the morbidly obese." Obesity Surgery, 14, 967969.

41. Roessner, V., Bleich, S., Banaschewski, T. and Rothenberger, A. (2005) "Olfactory deficits in anorexia nervosa." European Archives of Psychiatry and Clinical Neuroscience, 255, 6-9.

42.Rolls, E.T. (2005) "Taste, olfactory, and food texture processing in the brain, and the control of food intake." Physiology \& Behavior, 85, 45-56

43.Sauer, H., Mack, I., Kohler, S., Siegle, S., Rieber, N., Zipfel, S., Klosterhalfen, S., Ritze, Y., Otto, B., Bischoff, S.C. and Enck, P. (2011) "[The influence of 5-hydroxatryptophan on gastric emptying and the levels of cortisol and gastrointestinal hormones under different fatty acid conditions]." Zeitschrift für Gastroenterologie, 49, 1011

44.Schreder, T., Albrecht, J., Kleemann, A.M., Schöpf, V., Kopietz, R., Anzinger, A., Demmel, M., Linn, J., Pollatos, O. and Wiesmann, M. (2008) "Olfactory performance of patients with anorexia nervosa and healthy subjects in hunger and satiety." Rhinology, 46, 175-183.
45.Simchen, U., Koebnick, C., Hoyer, S., Issanchou, S. and Zunft, H.J. (2006) "Odour and taste sensitivity is associated with body weight and extent of misreporting of body weight." European Journal of Clinical Nutrition, 60, 698-705.

46.Stafford, L.D. and Welbeck, K. (2011) "High hunger state increases olfactory sensitivity to neutral but not food odors." Chemical Senses, 36, 189-198

47.Stehberg, J., Moraga-Amaro, R. and Simon, F. (2011) "The role of the insular cortex in taste function." Neurobiology of Learning and Memory, 96, 130-135.

48.Steinbach, G.C., Macias, B.R., Tanaka, K., Yost, W.T. and Hargens, A.R. (2005) "Intracranial pressure dynamics assessed by noninvasive ultrasound during 30 days of bed rest." Aviation, Space, Environmental Medicine, 76, 85-90.

49.Steinbach, S., Reindl, W., Kessel, C., Ott, R., Zahnert, T., Hundt, W., Heinrich, P., Saur, D. and Huber, W. (2010) "Olfactory and gustatory function in irritable bowel syndrome." European Archives of Otorhinolaryngology, 267, 1081-1087.

50.Teufel, M., Rieber, N., Meile, T., Giel, K.E., Sauer, H., Hünnemeyer, K., Enck, P. and Zipfel, S. (2012) "Body image after sleeve gastrectomy: reduced dissatisfaction and increased dynamics." Obesity Surgery, 22, 1232-1237.

51.Tong, J., Mannea, E., Aimé, P., Pfluger, P.T., Yi, C.X., Castaneda, T.R., Davis, H.W., Ren, X., Pixley, S., Benoit, S., Julliard, K., Woods, S.C., Horvath, T.L., Sleeman, M.M., D'Alessio, D., Obici, S., Frank, R. and Tschöp, M.H. (2011) "Ghrelin enhances olfactory sensitivity and exploratory sniffing in rodents and humans." Journal of Neuroscience, 31, 5841-5846.

52.Trappe, S.W., Trappe, T.A., Lee, G.A., Widrick, J.J., Costill, D.L. and Fitts, R.H. (2001). "Comparison of a space shuttle flight (STS-78) and bed rest on human muscle function." Journal of Applied Physiology, 91, 57-64.

53.Trappe, T., Trappe, S., Lee, G., Widrick, J., Fitts, R., \& Costill, D. (2006) "Cardiorespiratory responses to physical work during and following 17 days of bed rest and spaceflight." Journal of Applied 
Physiology, 100, 951-957

54.Turetsky, B.I., Hahn, C.G., BorgmannWinter, K. and Moberg, P.J. (2009) "Scents and nonsense: olfactory dysfunction in schizophrenia." Schizophrenia Bulletin, 35, 1117-1131.

55.Vickers, Z.M., Rice, B.L., Rose, M.S. and Lane, H.W. (2001) "Simulated microgravity [bed rest] has little influence on taste, odor or trigeminal sensitivity." Journal of Sensory Studies, 16, 23-32.

56.Wang, B.L., Takigawa, T., Yamasaki, Y., Sakano, N., Wang, D.H. and Ogino, K. (2008) "Symptom definitions for SBS (sick building syndrome) in residential dwellings."
International Journal of Hygiene and Environmental Health, 211, 114-120.

57.Wiesmann, M., Kopietz, R., Albrecht, J., Linn, J., Reime, U., Kara, E., Pollatos, O., Sakar, V., Anzinger, A., Fesl, G., Brückmann, H., Kobal, G. and Stephan, T. (2006) "Eye closure in darkness animates olfactory and gustatory cortical areas." Neuroimage, 32, 293-300.

58.Yeomans, M.R. and Mobini, S. (2006) "Hunger alters the expression of acquired hedonic but not sensory qualities of foodpaired odors in humans." Journal of Experimental Psychology. Animal Behavior Processes, 32, 460-466. 